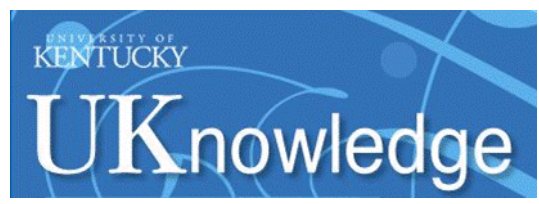

University of Kentucky

UKnowledge

\title{
Effect of Applied Magnetic Field on Shock Boundary Layer Interaction
}

Ovais U. Khan

University of Kentucky

Alexandre Martin

University of Kentucky, alexandre.martin@uky.edu

Follow this and additional works at: https://uknowledge.uky.edu/me_facpub

Part of the Aerodynamics and Fluid Mechanics Commons, and the Computer Sciences Commons Right click to open a feedback form in a new tab to let us know how this document benefits you.

\section{Repository Citation}

Khan, Ovais U. and Martin, Alexandre, "Effect of Applied Magnetic Field on Shock Boundary Layer Interaction" (2012). Mechanical Engineering Faculty Publications. 17.

https://uknowledge.uky.edu/me_facpub/17

This Conference Proceeding is brought to you for free and open access by the Mechanical Engineering at UKnowledge. It has been accepted for inclusion in Mechanical Engineering Faculty Publications by an authorized administrator of UKnowledge. For more information, please contact UKnowledge@lsv.uky.edu. 


\section{Effect of Applied Magnetic Field on Shock Boundary Layer Interaction}

Digital Object Identifier (DOI)

http://dx.doi.org/10.2514/6.2012-355

\section{Notes/Citation Information}

Published in the Proceedings of the 50th AIAA Aerospace Sciences Meeting including the New Horizons Forum and Aerospace Exposition, Paper 2012-0355, p. 1-12.

Copyright (@ 2012 by Ovais U. Khan and Alexandre Martin.

The copyright holders have granted the permission for posting the article here. 


\title{
Effect of Applied Magnetic Field on Shock Boundary Layer Interaction
}

\author{
Ovais U. Khan ${ }^{*}$ and Alexandre Martin ${ }^{\dagger}$ \\ Department of Mechanical Engineering \\ University of Kentucky, Lexington, $K Y$
}

\begin{abstract}
The governing magneto-hydrodynamic (MHD) equations contain classical fluid dynamics equations along with coupled Maxwell's magnetic induction equations. These equations model both advection and diffusion effects of electromagnetic field. However, available literature indicates that some previous investigations neglect the diffusion of magnetic field and considered only ideal MHD equations for modeling a typical MHD problem. In this work, the effects of magnetic field diffusion term also known as viscous magnetic term have been investigated over flow structure. Low magnetic Reynolds number approximation and ideal full MHD set of equations have been considered and solved using a four-stage modified Runge-Kutta scheme augmented with the Davis-Yee symmetric Total Variation Diminishing model in post-processing stage. Results obtained from viscous and ideal flow computations without applied magnetic field have been found in close agreement. However, results obtained from viscous MHD and ideal MHD computations substantially disagree from each other which indicate that the effect of magnetic diffusion term on overall flow structure is significant.
\end{abstract}

\section{Nomenclature}

$B_{0} \quad=$ magnetic field strength at stagnation point

$$
\begin{array}{lll}
\overrightarrow{\mathbf{B}}=\left\{\begin{array}{l}
B_{x} \\
B_{y} \\
B_{z}
\end{array}\right\} & =\text { magnetic field vector } \\
e_{t} & =\text { total energy per unit mass } \\
\mathbf{E} & =\text { convective flux vector in } x \text {-direction } \\
\mathbf{E}_{v} & =\text { diffusion flux vector in } x \text {-direction } \\
\mathbf{F} & =\text { convective flux-vector in } y \text {-direction } \\
\mathbf{F}_{v} & =\text { diffusion flux-vector in } y \text {-direction } \\
\overline{\mathbf{I}} & & =\text { identity tensor } \\
J & & \text { Jacobian of transformation } \\
\overrightarrow{\mathbf{J}} & & =\text { current density vector } \\
M_{\infty} & =\text { free stream Mach number } \\
p & =\text { pressure } \\
q & =\text { dynamic pressure } \\
\mathbf{Q} & =\text { field vector } \\
R_{m} & =\text { magnetic Reynolds number } \\
\mathbf{S}_{\text {MHD }} & =\text { source term in low } R_{m} \text { formulation } \\
t & =\text { time }
\end{array}
$$

\footnotetext{
* Research Associate, Member AIAA

${ }^{\dagger}$ Assistant Professor, Senior Member AIAA
} 


$$
\begin{aligned}
& \overrightarrow{\mathbf{U}}=\left\{\begin{array}{l}
u \\
v \\
w
\end{array}\right\}=\text { velocity vector } \\
& x, y=\text { Cartesian coordinates } \\
& \gamma \quad=\text { ratio of specific heats } \\
& \eta, \xi=\text { generalized coordinate } \\
& \eta_{x}, \eta_{y} \quad=\text { transformation metrics } \\
& \mu_{e o} \quad=\text { free space magnetic permeability } \\
& \rho \quad=\text { density } \\
& \sigma_{e} \quad=\text { electrical conductivity } \\
& \xi_{x}, \xi_{y} \quad=\text { transformation metrics } \\
& \text { subscripts } \\
& \infty=\text { free stream condition } \\
& \text { ref }=\text { reference or free stream condition } \\
& e \quad=\text { electromagnetic quantity } \\
& v \quad=\text { diffusion quantity } \\
& \text { superscripts } \\
& n=\text { iteration (time) level } \\
& \text { indices } \\
& i \quad=\text { index in } \xi \text {-direction } \\
& j \quad=\text { index in } \eta \text {-direction }
\end{aligned}
$$

\section{Introduction}

$\mathrm{H}^{\prime}$ IGH-SPEED flow over a compression corner has many applications with respect to future hypersonic flight where fluid properties such as pressure, temperature and density change abruptly as they cross the shock. Extremely complex flow field observed in a hypersonic flow regime involves high temperature gradients and chemical reactions. Magneto-Hydrodynamics (MHD) control of flow is one of the important techniques available amongst various flow control technologies. In fact, recent developments in superconducting materials and improvements in artificial ionization techniques have resulted in consideration of electromagnetic field as a tool for modifying heat transfer rates, drag, skin friction and shockwave boundary layer interaction for hypersonic flow regime.

Several studies have been devoted to understanding MHD high-speed flows. Shang et al. ${ }^{1}$ used ideal MHD equations for infinitely conducting fluids. They utilized finite volume numerical method for modeling the flow over two-dimensional cylindrical nose blunt body and predicted that application of magnetic field not only causes an increase in shock stand off distance, but also generates a secondary shock wave in the stagnation region.

Gaitonde and Poggie ${ }^{2}$ used full MHD equations of finitely conducting fluid to simulate inviscid flow over twodimensional cylindrical body with non-uniform magnetic field distribution. They identified fluid electrical conductivity as one of the most critical parameters for enhancing the interaction between fluid and magnetic field.

The low magnetic Reynolds number approach was utilized by Poggie and Gaitonde ${ }^{3}$ to model viscous and inviscid flows over a hemisphere. They showed that the application of magnetic field was causing an increase in shock stand off distance for both viscous and inviscid flows and concluded that qualitative changes in pressure field obtained by applying magnetic field were negligibly affected by viscous effects for simple blunt body configurations.

Damevin et al. ${ }^{4}$ utilized ideal MHD equations under the assumption of infinitely conducting fluid. Increase in shock stand off distance with the formation of secondary expansion wave and decrease in surface pressure were observed with the application of magnetic field.

In Ref. 5, Khan et al. explored the aspects of different types of magnetic field distribution under the low magnetic Reynolds number approximation for two-dimensional inviscid high speed flows. It was found that electromagnetic forces caused flow compression in the post shock region. They concluded that maximum stand off distance for a shock can be obtained with vortex type of magnetic field distribution. 
Conservation form of ideal MHD equations for modeling supersonic flow of real gas in equilibrium over a blunt body was used by MacCormack ${ }^{6}$. Implicit modified algorithm based on finite volume approach of discretization was implemented. Bow shock wave displacement towards upstream region and reversal of magnetic field near the exit section at the body shoulder was observed for the MHD computation.

MacCormack ${ }^{7}$ further investigated viscous MHD flow over the surface of an axisymmetric sphere/cone with dipole type of magnetic field placed at the center of sphere. Supersonic flow of real gas in equilibrium and hypersonic flow of nonequilibrium gases with chemical and thermal effects were considered. Different types of boundary conditions for magnetic field at the body surface have been implemented. For the boundary condition of zero normal derivatives of all magnetic components at the body surface, a decrease in heat transfer rate, decrease in velocity gradient (skin friction) and increase in total drag with the increase of magnetic field strength for isothermal wall boundary condition was noted. In the case of applied magnetic field at the wall, less interaction between plasma and magnetic field was achieved.

Lee, Huerta and Zha ${ }^{8}$ investigated hypersonic blunt body flow using low magnetic Reynolds number approach. They considered chemically equilibrium and chemically frozen states of gas over a hemispherical blunt body configuration. For MHD analysis, a dipoler type of magnetic field distribution is generated by placing a dipole at the body center. An increase in shock stand off distance and decrease in body surface temperature are observed after the application of magnetic field. It has been reported that increase in shock stand off distance and decrease in temperature levels were relatively less when chemical equilibrium condition is considered.

In Ref. 9, Bisek et al. introduced the MHD effects into an unstructured three-dimensional fluid dynamic code named LeMANS for investigating plasma-assisted hypersonic flow regime. MHD terms have been added to the conservation form of Navier-Stokes equations for a perfect gas flow under low magnetic Reynolds number approximation. Several electrical conductivity models have been implemented including Boltzmann's model. The developed MHD model has been validated with the existing closed form solution with Dirichlet boundary conditions and flow between two electrode plates with a potential difference.

Recently, Bisek et al. ${ }^{10}$ investigated MHD hypersonic flow over a hemisphere-capped cylinder with improved electrical conductivity model. Dipolar type of magnetic field is generated by placing a magnet at the center of the body. Increase in shock stand off and decrease in heat transfer at the stagnation portion is observed, however, a slight increase in heating at aft section of the body caused increase in total heat to the geometry. They reported that the results obtained with newly developed electrical conductivity model, closely match with the experimental data available in the literature.

Available literature indicates that some research efforts have considered ideal form of MHD equations for modeling a typical MHD flow field; for example, Ref. 1, 4 and 6. However, present investigation indicates that omission of magnetic viscous effects for modeling MHD flow may not provide correct results in regions of shock boundary layer interaction. In present work, comparison of results obtained from viscous and ideal MHD solvers have been presented for simulating flow over compression and expansion ramps.

\section{Basic Governing Equations}

The basic governing equations for modeling MHD flow consist of fluid dynamics equation coupled with Maxwell's magnetic induction equations. For a neutrally conducting medium, MHD equations termed as full MHD equations are summarized below:

\section{A.Full MHD Equations}

Full MHD equations involve coupled magnetic field equation along with fluid dynamics equations that also contain magnetic terms such as magnetic pressure and advection in momentum and energy equations. These equations in vector form can be expressed as

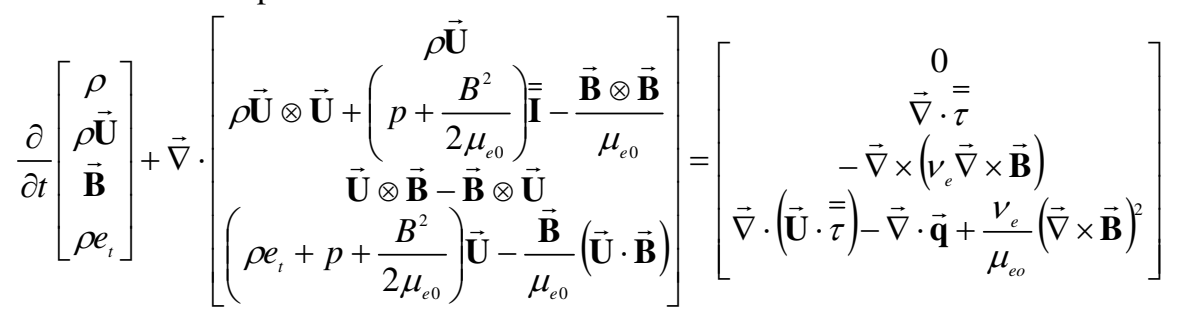




$$
\rho e_{t}=\frac{1}{2} \rho U^{2}+\frac{p}{\gamma-1}+\frac{B^{2}}{2 \mu_{e 0}}
$$

The above set of equations contains continuity, momentum, energy and Maxwell's magnetic induction equations. By turning-off the magnetic field terms, conventional Navier-Stokes equations of fluid dynamics can be obtained.

Subsequently, these equations can be rearranged in compact flux-vector formulation and presented for a twodimensional flow field in Cartesian coordinates:

$$
\frac{\partial \mathbf{Q}}{\partial t}+\frac{\partial \mathbf{E}}{\partial x}+\frac{\partial \mathbf{F}}{\partial y}+\mathbf{H}=\frac{\partial \mathbf{E}_{v}}{\partial x}+\frac{\partial \mathbf{F}_{v}}{\partial y}
$$

where $\mathbf{Q}$ is the unknown flux vector

and

$$
\begin{gathered}
\mathbf{Q}=\left[\begin{array}{llllllll}
\rho & \rho u & \rho v & \rho w & B_{x} & B_{y} & B_{z} & \rho e_{t}
\end{array}\right]^{T} \\
\rho e_{t}=\frac{1}{2} \rho U^{2}+\frac{p}{\gamma-1}+\frac{B^{2}}{2}
\end{gathered}
$$

$\mathbf{E}, \mathbf{F}$ are the inviscid flux vectors, $\mathbf{E}_{v}, \mathbf{F}_{v}$ are diffusion flux vectors and $\mathbf{H}$ is the additional term. This additional term has been introduced based on Gauss' law of magnetism for overcoming the singularity associated with the flux Jacobian matrices. The details of additional term $\mathbf{H}$ and flux vectors are provided in Ref. 11 .

The above governing equations are known as full MHD equations in the available literature. From these equations, ideal MHD equations can be obtained by setting right-hand side of equation (3) equal to zero. That is, ideal MHD equation is

$$
\frac{\partial \mathbf{Q}}{\partial t}+\frac{\partial \mathbf{E}}{\partial x}+\frac{\partial \mathbf{F}}{\partial y}+\mathbf{H}=0
$$

In this equation the definitions of unknown flux vector $\mathbf{Q}$, inviscid flux vectors $\mathbf{E}, \mathbf{F}$ and additional term $\mathbf{H}$ will remain the same. Besides, full MHD set of equations there exists an approximate formulation known low magnetic Reynolds number approximation, for modeling restricted MHD flows. Details of this approach are provided in the next section.

\section{B.Low Magnetic Reynolds Number Approximation}

This formulation contains set of governing equations that approximate electromagnetic effects by adding source terms in the fluid dynamics equations. The governing equations for the unsteady flow of viscous fluid under low magnetic Reynolds number formulation are

$$
\frac{\partial \mathbf{Q}}{\partial t}+\frac{\partial \mathbf{E}}{\partial x}+\frac{\partial \mathbf{F}}{\partial y}=\frac{\partial \mathbf{E}_{v}}{\partial x}+\frac{\partial \mathbf{F}_{v}}{\partial y}+\boldsymbol{S}_{M H D}
$$

where $\mathbf{Q}$ is the unknown vector, $\mathbf{E}, \mathbf{F}, \mathbf{E}_{v}$ and $\mathbf{F}_{v}$ are the inviscid and viscous flux vectors. The additional source term is represented by $S_{M F D}$.

$$
\begin{aligned}
& \text { where } \quad \mathbf{Q}=\left[\begin{array}{lllll}
\rho & \rho u & \rho v & \rho w & \rho e_{t}
\end{array}\right]^{T} \\
& \text { with } \\
& \rho e_{t}=\frac{1}{2} \rho U^{2}+\frac{p}{\gamma-1}
\end{aligned}
$$

Details of inviscid $\mathbf{E}, \mathbf{F}$ and viscous $, \mathbf{E}_{v}, \mathbf{F}_{v}$ flux vectors and source term $\boldsymbol{S}_{\text {MHD }}$ are provided in Ref. 11. Moreover, equations of full MHD approach and low magnetic Reynolds number approximation have been nondimensionalized according to the procedure outlined in Ref. 11.

\section{Full MFD Equations}

\section{Generalized Coordinates}

The governing equations in physical space are transformed to a computational space for both modeling approaches. First, the nondimensionalized full MFD equation (3) in flux-vector formulation has been represented in generalized curvilinear coordinates as follows:

$$
\frac{\partial \overline{\mathbf{Q}}}{\partial t}+\frac{\partial \overline{\mathbf{E}}}{\partial \xi}+\frac{\partial \overline{\mathbf{F}}}{\partial \eta}+\overline{\mathbf{H}}=\frac{\partial \overline{\mathbf{E}}_{v}}{\partial \xi}+\frac{\partial \overline{\mathbf{F}}_{v}}{\partial \eta}
$$


where

$$
\overline{\mathbf{Q}}=\frac{\mathbf{Q}}{J}
$$

$\overline{\mathbf{E}}, \overline{\mathbf{F}}$, and $\overline{\mathbf{E}}_{v}, \overline{\mathbf{F}}_{v}$ represent the convective and diffusion flux vectors, respectively. The detail of each fluxvector has been provided in Ref. 11. The MFD equation (8) can be rewritten as follows by definition of the Jacobian matrices,

$$
\frac{\partial \overline{\mathbf{Q}}}{\partial t}+\overline{\mathbf{A}} \frac{\partial \overline{\mathbf{Q}}}{\partial \xi}+\overline{\mathbf{B}} \frac{\partial \overline{\mathbf{Q}}}{\partial \eta}=\frac{\partial \overline{\mathbf{E}}_{v}}{\partial \xi}+\frac{\partial \overline{\mathbf{F}}_{v}}{\partial \eta}
$$

where $\overline{\mathbf{A}}$ and $\overline{\mathbf{B}}$ are the flux Jacobian matrices, their eigenvalues represent the wave speeds in $\xi$ and $\eta$ directions respectively. Details of these transformation matrices have been discussed Ref. 11.

\section{Low Magnetic Reynolds Number Approach}

The governing equation (6) for the low magnetic Reynolds number approximation is transformed to a computational space and expressed as

$$
\frac{\partial \overline{\mathbf{Q}}}{\partial t}+\frac{\partial \overline{\mathbf{E}}}{\partial \xi}+\frac{\partial \overline{\mathbf{F}}}{\partial \eta}=\frac{\partial \overline{\mathbf{E}}_{v}}{\partial \xi}+\frac{\partial \overline{\mathbf{F}}_{v}}{\partial \eta}+\overline{\mathbf{S}}_{M F D}
$$

where

$$
\overline{\mathbf{S}}_{M F D}=\frac{1}{J} \mathbf{S}_{M F D}
$$

The MHD equation (10) can be rewritten as follows by definition of the Jacobian matrices,

$$
\frac{\partial \overline{\mathbf{Q}}}{\partial t}+\overline{\mathbf{A}} \frac{\partial \overline{\mathbf{Q}}}{\partial \xi}+\overline{\mathbf{B}} \frac{\partial \overline{\mathbf{Q}}}{\partial \eta}=\frac{\partial \overline{\mathbf{E}}_{v}}{\partial \xi}+\frac{\partial \overline{\mathbf{F}}_{v}}{\partial \eta}+\overline{\mathbf{S}}_{M F D}
$$

Details of transformation matrices $\overline{\mathbf{A}}$ and $\overline{\mathbf{B}}$ for low magnetic Reynolds number approximation can be found in Ref. 12. It is important to mention that flux Jacobian matrices $\overline{\mathbf{A}}$ and $\overline{\mathbf{B}}$ are simpler than those obtained from full MFD equation.

\section{Numerical Method}

The modified Runge-Kutta scheme stabilized with TVD scheme for damping the numerical oscillations associated with convective fluxes is used to obtain numerical solutions. The scheme can be expressed as

$$
\overline{\mathbf{Q}}_{i, j}^{(0)}=\overline{\mathbf{Q}}_{i, j}^{n}
$$

for $m=1$ to 4 and $\alpha_{m}=4$ to 1

where

$$
\overline{\mathbf{Q}}_{i, j}^{(m)}=\overline{\mathbf{Q}}_{i, j}^{n}-\frac{\Delta t}{\alpha_{m}} \bar{f}_{i, j}^{(m-1)}
$$

$$
\begin{array}{ll}
\bar{f}=\frac{\partial \overline{\mathbf{E}}}{\partial \xi}+\frac{\partial \overline{\mathbf{F}}}{\partial \eta}+\overline{\mathbf{H}}-\frac{\partial \overline{\mathbf{E}}_{v}}{\partial \xi}-\frac{\partial \overline{\mathbf{F}}_{v}}{\partial \eta}, & \text { for full MFD equations } \\
\bar{f}=\frac{\partial \overline{\mathbf{E}}}{\partial \xi}+\frac{\partial \overline{\mathbf{F}}}{\partial \eta}-\frac{\partial \overline{\mathbf{E}}_{v}}{\partial \xi}-\frac{\partial \overline{\mathbf{F}}_{v}}{\partial \eta}-\overline{\mathbf{S}}_{M F D}, & \text { for low } R_{\mathrm{m}} \text { approximation }
\end{array}
$$

Second-order central difference approximations have been utilized for the convective and diffusion terms according to the procedure outlined in Ref. 13. The post-processing stage is the last stage of computation and consists of correcting the last calculated unknown vector. Mathematically, it can be expressed as,

$$
\overline{\mathbf{Q}}_{i, j}^{n+1}=\overline{\mathbf{Q}}_{i, j}^{(4)}+\overline{\mathbf{T V D}}^{n}
$$

The TVD model is based on the eigenstructure of the convective flux Jacobian matrices. The eigenstructure and numerical method for general ideal MHD equations are provided in Ref. 13. For the low magnetic Reynolds number approximation, the eigenstructure and numerical method are the same as those used for Euler equations, which are also described in Ref. 13. 


\section{Results}

Supersonic flows over compression and expansion ramps have been considered for the present investigation. First, the flow field over a compression ramp is computed and results are presented in this section. The wedge angle for compression corner is set to 10 degrees with a ramp of 0.6 meter length; whereas, inlet section length is set equal to 0.4 meter. The computational grid system is shown in Figure 1; grid clustering has been implemented near the wall region to capture viscous effects accurately.

outflow

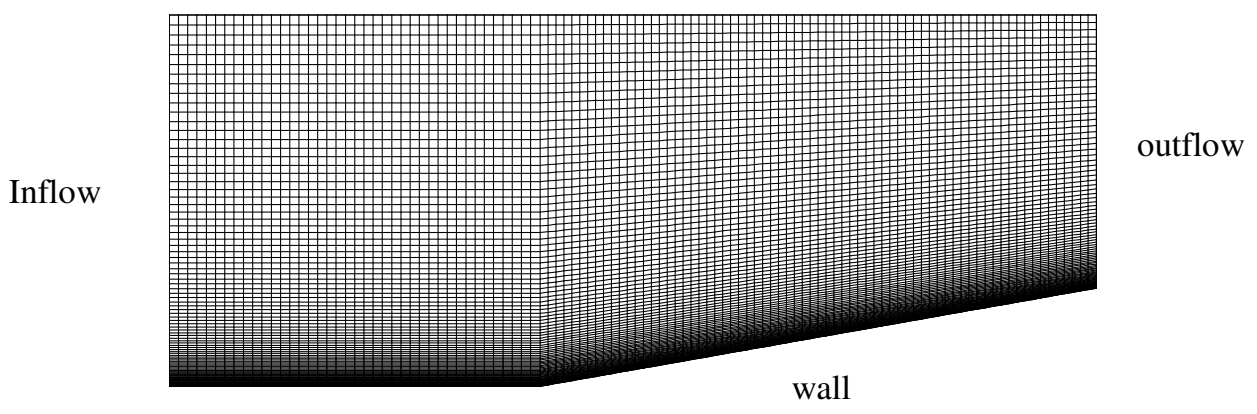

Figure 1: Computational grid used for present analysis.

For numerical simulation, free stream conditions are set according to the atmospheric conditions at an altitude of $40 \mathrm{~km}$ with a free stream Mach number of 10 as

\begin{tabular}{|l|l|}
\hline Pressure: & $p_{\infty}=277.52 \mathrm{~Pa}$ \\
\hline Temperature: & $T_{\infty}=251.05 \mathrm{~K}$ \\
\hline Mach number & $M_{\infty}=10.0$ \\
\hline
\end{tabular}

The boundary conditions are specified as:

$\begin{array}{ll}\text { Wall } & \left\{\begin{array}{l}\text { no - slip/slip condition } \\ \text { zero pressure gradient } \\ \text { adiabatic }\end{array}\right. \\ \text { Inflow } & \left\{\begin{array}{l}\text { free stream conditions } \\ \text { zero magnetic field }\end{array}\right.\end{array}$

Outflow \{zero - order extrapolation for all primitive variables

Uniform magnetic field along the y-direction is applied for MHD computation. The results of compression corner analysis are summarized in Figs 2 through 9.

Figure 2 illustrates the pressure pattern for Mach 10 flow over the compression ramp for the Navier-Stokes analysis. Formation of an oblique shock wave at the corner is evident. Figure 3 indicates the pressure contours for the inviscid flow computation. A similar shock wave angle is noted; however, absence of viscous boundary layer is also evident for this case.

For MHD analysis, pressure contours with applied uniform magnetic field of strength $0.025 \mathrm{~T}$ along the $y$ direction are shown in Fig. 4 for the viscous MHD computation; the corresponding magnetic Reynolds number is 0.0379. The Lorentz force has caused an increase in the shock-wave angle and displacement of shock-wave towards the inlet section. Moreover, shock thickening occurred after the application of magnetic field, is also obvious in this figure.

Figure 5 shows pressure contours obtained from the ideal MHD calculations. It is interesting to observe that the applied magnetic field not only causes an increase in shock-wave angle but also results in formation of a secondary wave. The two (viscous and ideal) MHD solutions differ substantially as evident from the figures, for viscous MHD computation (Fig. 4), application of magnetic field has caused a significant movement of shock towards the inlet 
section and no secondary shock-wave is observed. However, for ideal MHD analysis (Fig. 5), a slight movement of shock towards the inlet section is occurred and a secondary shock-wave is observed after the application of electromagnetic field. Further investigation of pressure field for the ideal MHD analysis, shows an increase in pressure after crossing the primary shock-wave; whereas, a significant drop in pressure values is observed after crossing the secondary shock-wave. Thus, it can be inferred that the secondary shock-wave is actually an expansionwave.

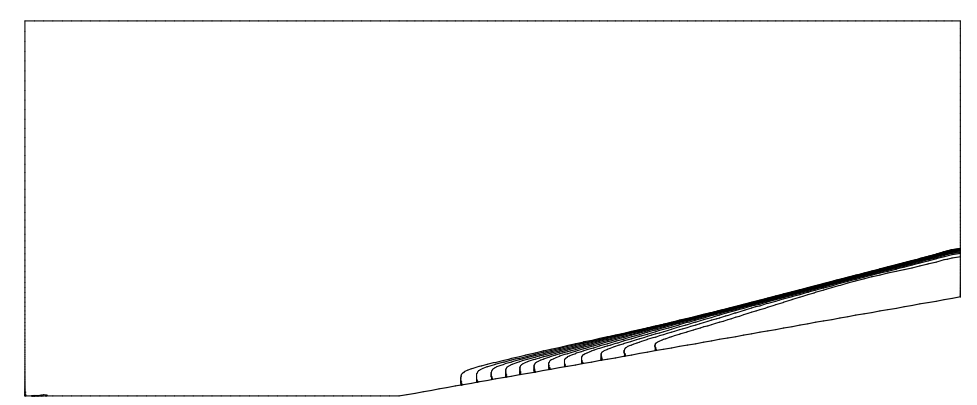

Figure 2: Pressure contours for the Navier-Stokes computation.

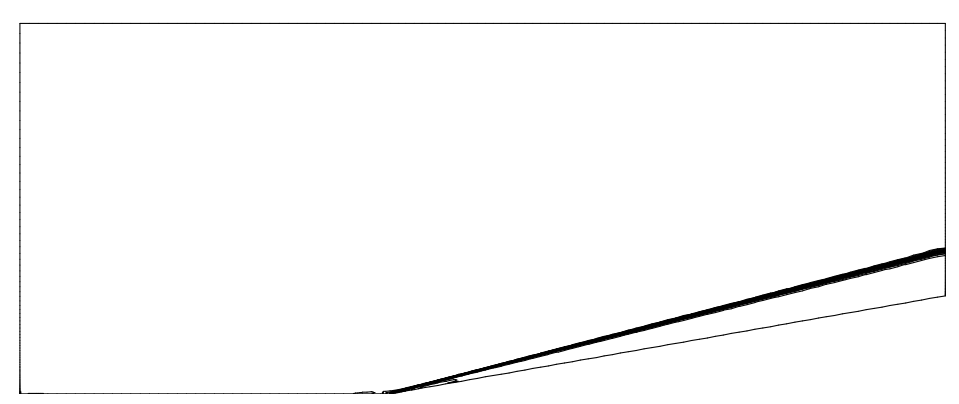

Figure 3: Pressure contours for ideal flow computation.

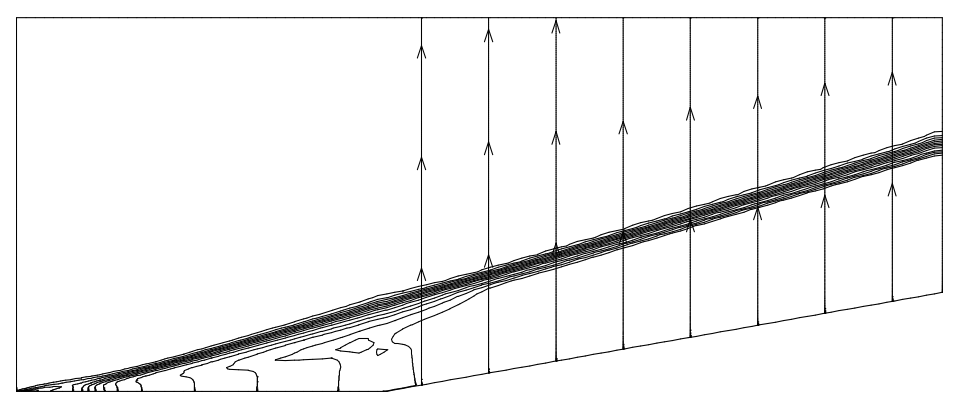

Figure 4: Pressure contours for the viscous MHD computation with By $=0.025 \mathrm{~T}$.

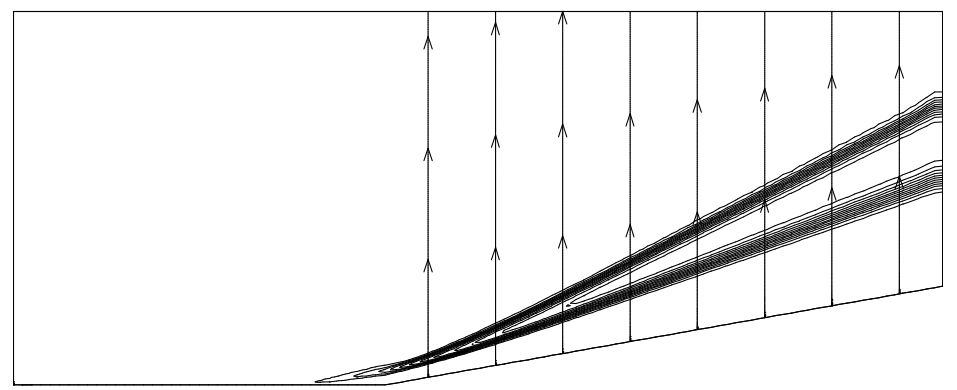

Figure 5: Pressure contours for the ideal MHD computation with $\mathrm{By}=0.025 \mathrm{~T}$.

American Institute of Aeronautics and Astronautics 
Furthermore, a comparison of velocity streamlines has also been made for the viscous and ideal flow computations without and with the application of magnetic field. Figure 6 shows the velocity streamlines for the Navier-Stokes analysis, formation of a circulation region at the ramp corner is obvious. For viscous MHD analysis, the Lorentz force generated after the application of magnetic field; has resulted in an increase in the size of circulation zone as depicted in Fig. 7.

Similarly, Fig. 8 illustrates the streamline patterns for the ideal flow computation without the application of magnetic field. As expected, streamlines are depicting the absence of boundary layer at the near wall region. The application of magnetic field has changed the streamline patterns in overall flow region as shown in Fig. 9; however, MHD interaction at the near wall region cannot be explored for this case due to the absence of viscous effects.

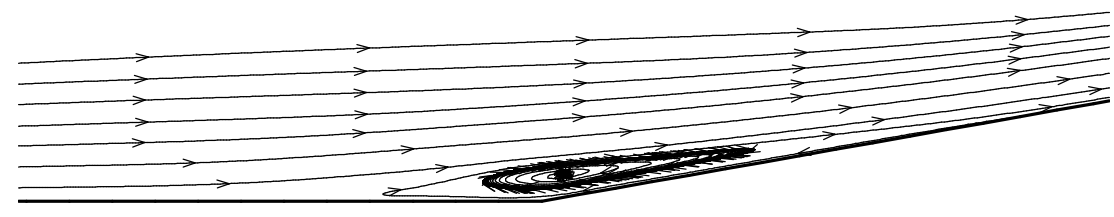

Figure 6: Velocity streamlines for the Navier-Stokes analysis.

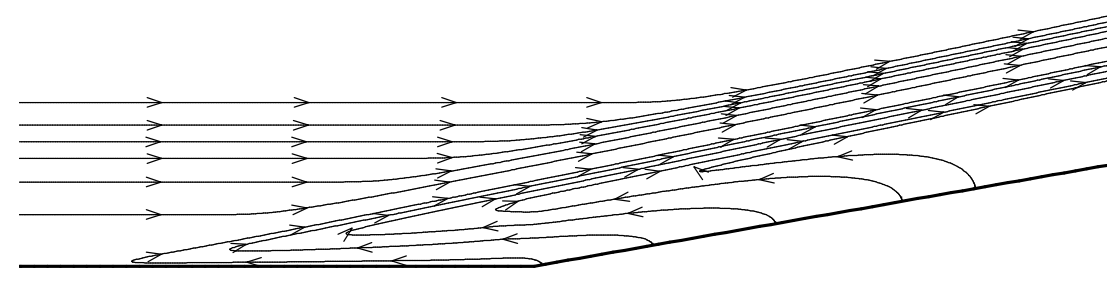

Figure 7: Velocity streamlines for the viscous MHD analysis with By $=0.025 \mathrm{~T}$.

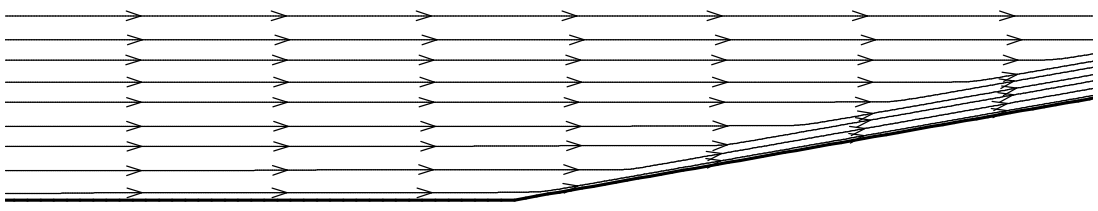

Figure 8: Velocity streamlines for the ideal flow analysis.

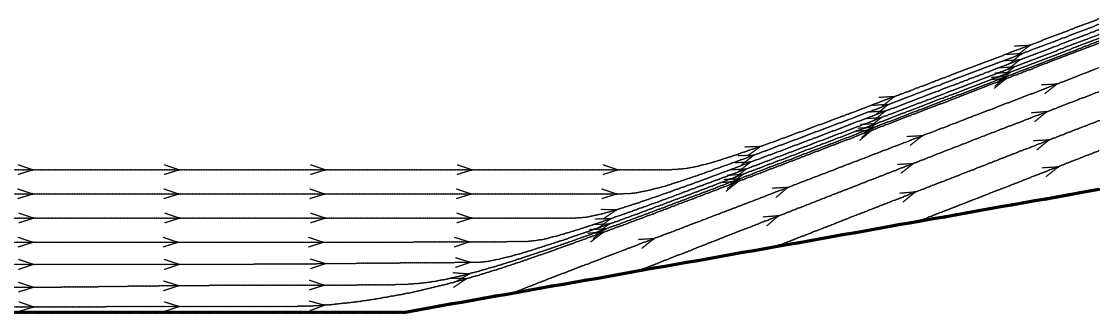

Figure 9: Velocity streamlines for the ideal MHD analysis with $\mathrm{By}=0.025 \mathrm{~T}$. 
Subsequently, flow over an expansion corner has been considered as a second case for investigating the magnetic viscous effects over flow field. The expansion corner angle is set to 10 degrees, length of ramp is 0.6 meter and inlet section length is set equal to 0.4 meter. The computational grid system is shown in Figure 10.

Free stream conditions have been set according to the previous case of compression corner at an altitude of $40 \mathrm{~km}$ with a free stream Mach number of 10. At the wall, no-slip boundary conditions for the velocity components, zeroorder extrapolation for the pressure and adiabatic condition for the temperature have been imposed. Zero-order extrapolation for all primitive variables is implemented at the outflow boundaries.

Figure 11 shows pressure contours for the viscous fluid dynamics computation of supersonic flow over an expansion corner. As expected, an expansion fan is observed in this flow field; however, the Mach lines are not converging at the corner because of boundary layer existence.

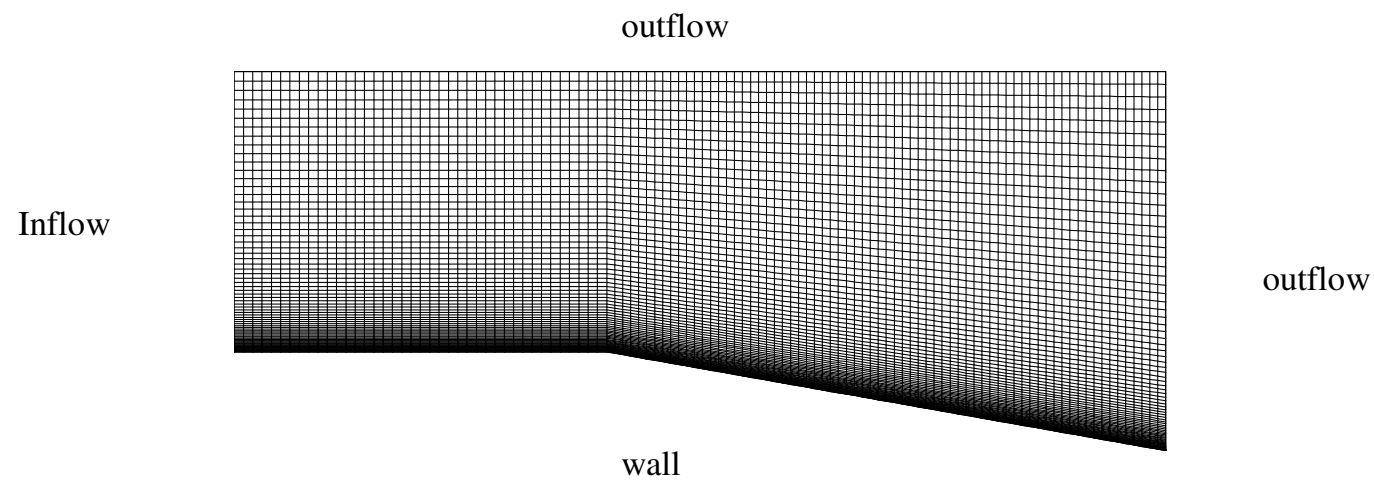

Figure 10: Computational grid used for expansion corner analysis.

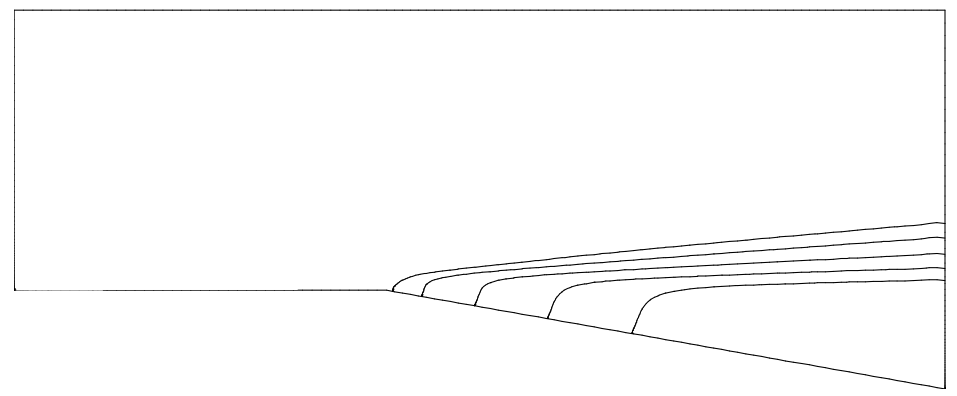

Figure 11: Pressure contours for the Navier-Stokes analysis of expansion corner.

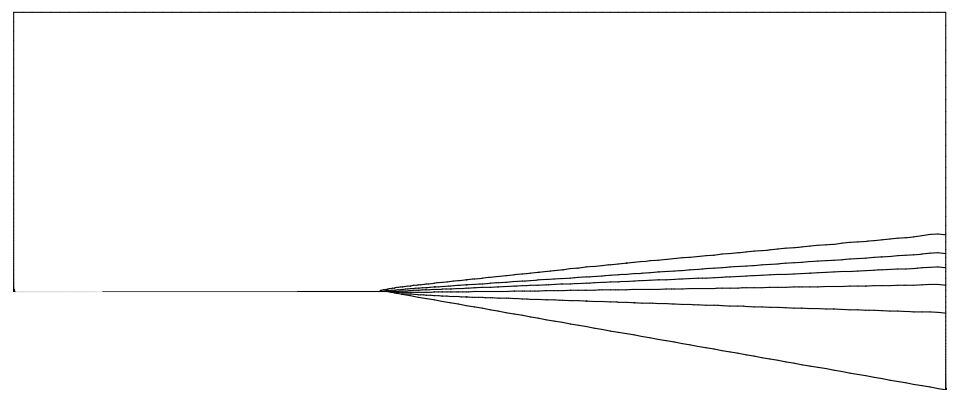

Figure 12: Pressure contours for ideal flow analysis of expansion corner. 
Computations for the similar operating conditions with the assumption of an inviscid flow field are also performed and corresponding pressure contours are presented in Fig. 12. Formation of an expansion fan is apparent with a converging trend in Mach lines near the corner region in contrast to the viscous flow field which is primarily due to absence of viscous effects. Thus, it can be concluded that the viscous effects for supersonic/hypersonic flow regimes over expansion corner significantly influence the flow parameters and affect merging of Mach lines at the corner.

Subsequent to performing the viscous and ideal fluid dynamic analyses without magnetic field application, MHD computations are executed with a uniform imposed magnetic field of $0.025 \mathrm{~T}$ along y-direction for both viscous and ideal MHD flow conditions. For viscous MHD analysis, Lorentz force has caused an increase in Mach wave angle and movement of Mach lines beyond the expansion corner as evident in Fig. 13. Moreover, coalescence in Mach waves is also observed after the application of magnetic field, this conjoining of Mach waves indicates that the Lorentz force has resulted in compression of the flow field for expansion ramp.

For ideal MHD analysis, pressure contours obtained from a converged solution are shown in Fig. 14. It is important to note that the Lorentz force has caused significant compression of flow field resulting in formation of primary and secondary shock-waves. A comparison between ideal and viscous MHD results for expansion corner flow field shows that both solutions significantly differ from each other. Additional probe of pressure field for the ideal MHD calculation reveals that pressure is increased after crossing the primary (upper) shock-wave; however, a substantial decrease in pressure values is observed after crossing the secondary (lower) shock-wave. Thus, indicating that the secondary shock-wave is an expansion-wave. It is interesting to note that pressure fields for compression and expansion ramps obtained from ideal MHD analysis have similar characteristic behavior. Results obtained from ideal MHD analysis suggest that magnetic viscous terms have dominant effects and their omission may not provide correct solution.

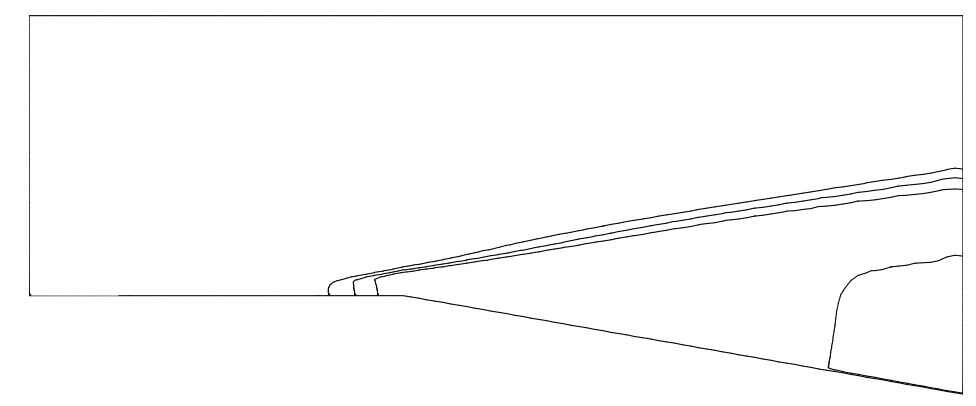

Figure 13: Pressure contours for viscous MHD flow analysis of expansion corner with By $=0.025 \mathrm{~T}$.

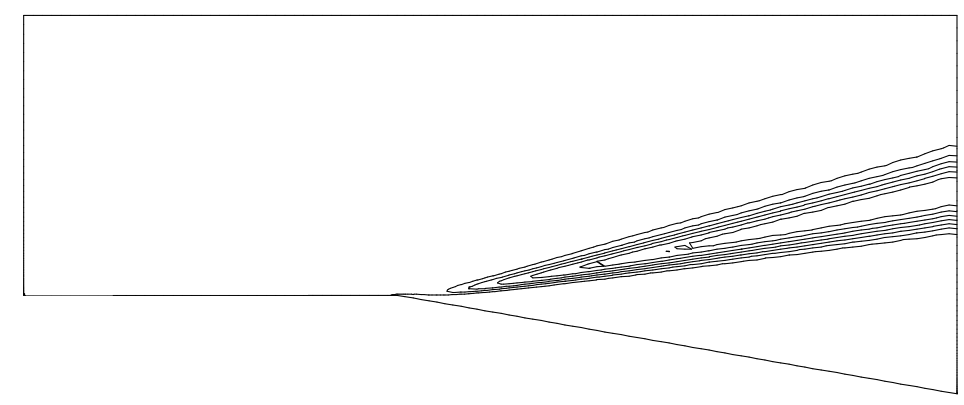

Figure 14: Pressure contours for ideal MHD flow analysis of expansion corner with $\mathrm{By}=0.025 \mathrm{~T}$.

Finally, velocity streamlines have been presented for viscous and ideal flow fields with and without the application of magnetic field. Fig. 15 illustrates the velocity streamlines for the Navier-Stokes computation; a deflecting trend in streamlines towards the downward direction is evident due to formation of expansion fan at the corner. Later, a uniform magnetic field of $0.025 \mathrm{~T}$ is imposed along the $\mathrm{y}$-direction for performing viscous MHD analysis, the corresponding velocity streamlines are shown in Fig. 16. Bending of stream traces towards the upper direction with slight shriveling confirms the flow compression phenomenon at the expansion ramp after the application of magnetic field. 
Moreover, velocity streamlines for the inviscid flow field with and without imposed magnetic field are also drawn. Figure 17 shows streamline patterns for the ideal flow computation without the application of magnetic field. As anticipated, streamlines have been deflected after crossing the expansion fan occurred at the corner. The stream traces are smoother for this flow field as compared to the stream traces obtained from the Navier-Stokes analysis. For ideal MHD analysis, an overall change in streamlines is obvious as shown in Fig. 18. Flow compression is substantial as compared to the viscous MHD effects; however, MHD interaction at the near wall region cannot be explored due to the absence of viscous effects.

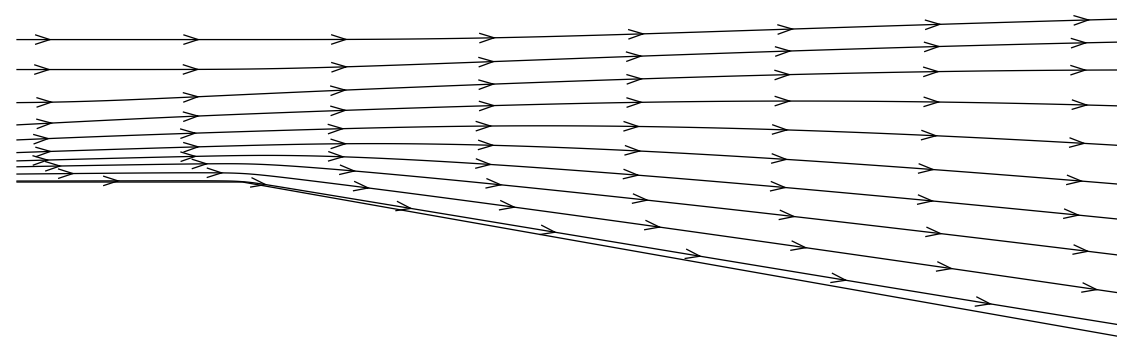

Figure 15: Velocity streamlines for the Navier-Stokes analysis.

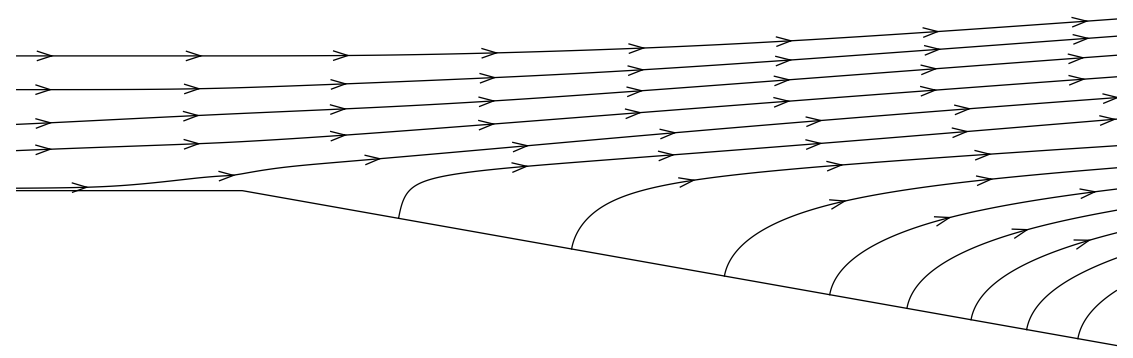

Figure 16: Velocity streamlines for the viscous MHD analysis with $B y=0.025 \mathrm{~T}$.

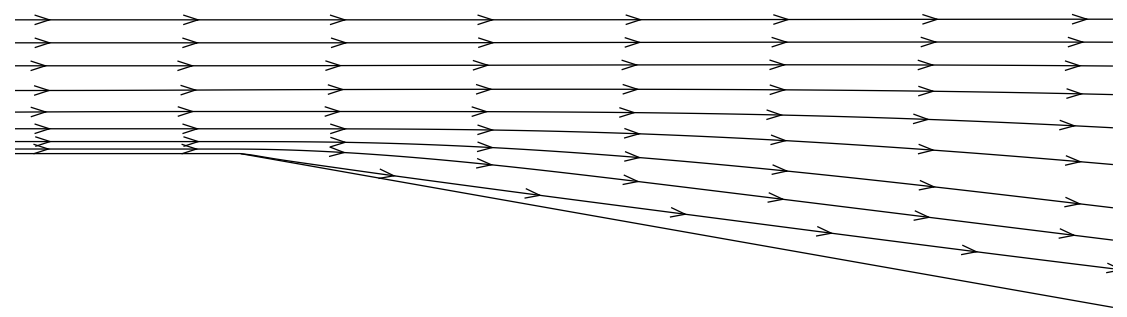

Figure 17: Velocity streamlines for the ideal flow analysis.

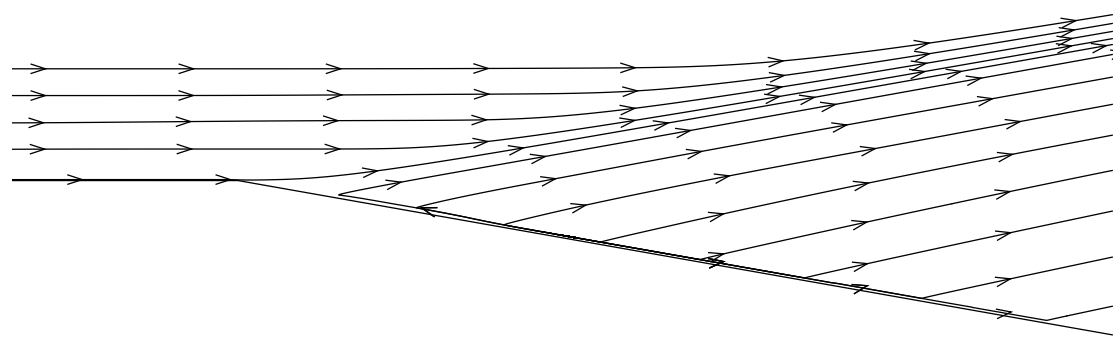

Figure 18: Velocity streamlines for the ideal MHD analysis with $\mathrm{By}=0.025 \mathrm{~T}$. 


\section{Conclusion}

Flow over compression and expansion ramps has been considered with and without the application of magnetic field. The effects of viscous terms on overall flow structure have been investigated before and after imposing the magnetic field. For compression ramp flow, results indicate that viscous and ideal flow solvers provide similar results in the absence of applied magnetic field. However, when magnetic field is turned on, results obtained from viscous MHD and ideal MHD solvers differ substantially. That is, for viscous MHD analysis of compression corner, the applied magnetic field has caused displacement of shock-wave and increase in shock-wave angle; however, for ideal MHD analysis, slight displacement of shock-wave with formation of a secondary shock-wave is observed after the application of magnetic field. In contrast to compression corner flow, presence of boundary layer significantly affects the flow structure for expansion ramp flow regime even before the application of magnetic field. That is, for the Navier-Stokes analysis dispersed Mach lines are obtained at region near the expansion corner; however, for inviscid analysis convergence in Mach lines is observed near the expansion corner region. Likewise, results obtained from viscous MHD and ideal MHD calculations significantly disagree from each other. For viscous MHD analysis, application of magnetic field has caused merging of Mach lines, thereby, causing flow compression at the expansion ramp. Nonetheless, for ideal MHD computation, a strong shock-wave followed by a primary shock-wave is noticed. Results obtained from compression and expansion corner flow regimes, suggest that the effects of magnetic diffusion term are significant and its elimination may not provide correct solution. Moreover, velocity stream patterns obtained from viscous and ideal MHD analyses of compression and expansion ramps indicate that the effects of viscous magnetic term are essential when investigating the influence of applied electromagnetic field for flow regime having low values of magnetic Reynolds number.

\section{Acknowledgments}

This research was supported by the US National Science Foundation through TeraGrid startup allocation resources, under grant CHE110061, and by the VP of Research Office and College of Engineering at the University of Kentucky. The authors would like to thank the University of Kentucky High Performance Computing Center for their assistance, as well as Dr. Klaus Hoffmann at Wichita State University for several useful discussions.

\section{References}

1. Shang, J. S., Canupp, P. W., and Gaitonde, D. V., "Computational Magneto-Aerodynamics Hypersonics", AIAA-1999-4903, 1999.

2. Gaitonde, D. V., and Poggie, J., "Simulations of Magnetogasdynamics Flow Control Techniques", AIAA-20002326, 2000.

3. Poggie, J., and Gaitonde, D. V., "Computational Studies of Magnetic Control in Hypersonic Flow", AIAA-20010196, 2001.

4. Damevin, H. M., Hoffmann, K. A., and Dietiker, J. F., "Numerical Simulation of Hypersonic MHD Application", AIAA-99-3611, 1999.

5. Khan, O. U., Hoffmann, K. A., and Dietiker, J. F., "Numerical Investigation of Magnetogasdynamic High Speed Flows", AIAA-2005-1182, 2005.

6. MacCormack, R. W., "An Upwind Conservation Form Method for the Ideal Magnetohydrodynamics Equations", AIAA-1999-3609, 1999.

7. MacCormack, R. W., “A Conservation Form Method for Magneto-Fluid Dynamics”, AIAA-2001-0195, 2001.

8. Lee, J., Huerta, M. A., and Zha, G., "Low Rem 3D MHD Hypersonic Equilibrium Flow Using High Order WENO Schemes", AIAA-2010-229, 2010.

9. Bisek, N. J., Boyd, I. D., and Poggie, J., "Numerical Study of Electromagnetic Aerodynamic Control of Hypersonic Vehicles", AIAA-2009-1000, 2009.

10. Bisek, N. J., Boyd, I. D., and Poggie, J., "Numerical Study of Magnetohydrodynamic Flow Around a Hemisphere", AIAA Journal of Spacecraft and Rockets, Vol. 47, No. 5, pp816-827, 2010.

11. Khan, O. U., Hoffmann, K. A., and Dietiker, J. F., "Validity of Low Magnetic Reynolds Number Formulation of Magnetofluiddynamic", AIAA-2007-4374, 2007.

12.Hoffmann, K. A., and Chiang, S. T., Computational Fluid Dynamics, Vol.II, $4^{\text {th }}$ ed., EES, Wichita, Kansas, 2000.

13.Harada, S., Augustinus, J., Hoffmann, K. A., and Agarawal, R. K., "Development of a Modified Runge-Kutta Scheme with TVD Limiters for the Ideal 1-D MHD Equations", AIAA-97-2090, June, 1997. 